\title{
Validation of quick sequential organ failure assessment score for poor outcome prediction among emergency department patients with suspected infection
}

eISSN: $2383-4625$

Received: 24 August 2018

Revised: 10 September 2018

Young Wha Sohn ${ }^{1}$, Hye Young Jang ${ }^{1}$, Suyeon Park' ${ }^{2}$, Youngjoo Lee', Young Shin Cho', Junbum Park', Heajin Chung', Sang-II Kim'

'Department of Emergency Medicine, Soonchunhyang University Seoul Hospital, Seoul, Korea

${ }^{2}$ Department of Biostatistics, Soonchunhyang University Seoul Hospital, Seoul, Korea

Objective The quick sequential organ failure assessment (qSOFA) score, which includes mentation, systolic blood pressure, and respiratory rate, was developed to identify serious sepsis in outof-hospital or emergency department (ED) settings. We evaluated the ability of the qSOFA score to predict poor outcome in South Korean ED patients with suspected infection.

Methods The qSOFA score was calculated for adult ED patients with suspected infection. Patients who received intravenous or oral antibiotics in the ED were considered to have infection. In-hospital mortality rate, admission rate, intensive care unit (ICU) admission rate, length of hospital stay (LOS), and lactate levels were compared between the qSOFA score groups. Receiver operating characteristic curves and area under the receiver operating characteristic curve values for in-hospital mortality were calculated according to qSOFA cut-off points and lactate levels.

Results Of 2,698 patients, in-hospital mortality occurred in 134 (5.0\%). The mortality rate increased with increasing qSOFA score $(2.2 \%, 6.4 \%, 17.5 \%$, and $42.4 \%$ for qSOFA scores $0,1,2$, and 3 , respectively, $P<0.001)$. The admission rate, ICU admission rate, $L O S$, and lactate level also increased with increasing qSOFA score (all $\mathrm{P}<0.001$ ). The area under the receiver operating characteristic curve values for predicting in-hospital mortality associated with qSOFA score, lactate $\geq 2 \mathrm{mmol} / \mathrm{L}$, and lactate $\geq 4 \mathrm{mmol} / \mathrm{L}$ were 0.719 (95\% confidence interval $[\mathrm{Cl}], 0.670$ to 0.768 ), $0.657(95 \% \mathrm{Cl}, 0.603$ to 0.710$)$, and $0.632(95 \% \mathrm{Cl}, 0.571$ to 0.693$)$, respectively.

Conclusion Patients with a higher qSOFA score had higher admission, ICU admission, and inhospital mortality rates, longer LOS, and higher lactate level. The qSOFA score showed better performance for predicting poor outcome than lactate level.

Keywords Hospital mortality; Sepsis; Lactate acid

Correspondence to: Hye Young Jang Department of Emergency Medicine, Soonchunyang University Seoul Hospital, 59 Daesagwan-ro, Yongsangu, Seoul 04401, Korea

E-mail: emedhy@schmc.ac.kr ORCID

http://orcid.org/0000-0002-2733-003X

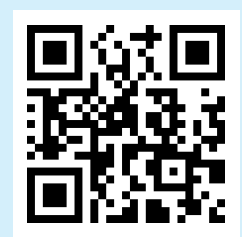

How to cite this article:

Sohn YW, Jang HY, Park S, Lee Y, Cho YS, Park J, Chung H, Kim SI. Validation of quick sequential organ failure assessment score for poor outcome prediction among emergency department patients with suspected infection. Clin Exp Emerg Med 2019;6(4):314-320.
This is an Open Access article distributed under the terms of the Creative Commons Attribution Non-Commercial License (http:// creativecommons.org/licenses/by-nc/4.0/). 


Capsule $\begin{aligned} & \text { What is already known } \\ & \text { The quick sequential organ failure assessment (qSOFA) score was developed to identify serious sepsis for out of hospital } \\ & \text { situation or the emergency department. } \\ & \text { What is new in the current study } \\ & \text { This study evaluated how adequately qSOFA score can predict poor outcome of patients with suspected infection in the } \\ & \text { emergency department setting in South Korea. Higher qSOFA score patients had higher admission rate, intensive care } \\ & \text { unit admission rate, in- hospital mortality rate, longer hospital stay and lactate level. }\end{aligned}$

\section{INTRODUCTION}

In 2016, an international task force convened by the Society of Critical Care Medicine and the European Society of Intensive Care Medicine newly defined sepsis as life-threatening organ dysfunction caused by a dysregulated host response to infection, and strongly recommended administration of antibiotics within 1 hour after recognition of sepsis. ${ }^{1}$ Mortality increases with delayed antimicrobial treatment. ${ }^{2}$

The sequential organ failure assessment (SOFA) score has been used to determine the extent of a person's organ function or rate of failure. ${ }^{1}$ However, since the SOFA requires laboratory results that take time, it is not adequate for out-of-hospital or emergency department (ED) use. For these purposes, the quick sequential organ failure assessment (qSOFA) was developed in 2016. ${ }^{1}$ The qSOFA includes altered mentation, systolic blood pressure of 100 $\mathrm{mmHg}$ or less, and respiratory rate of $22 / \mathrm{min}$ or greater. Each criterion is assigned 1 point, and organ dysfunction is suspected for scores of 2 and higher. The qSOFA can be calculated rapidly in the ED without need for laboratory or advanced testing, which makes it useful in emergency settings.

Since most unstable patients, including those with sepsis, come through the emergency department, it is important for the emergency physician to recognize these patients as soon as possible and start resuscitation. Faster recognition of sepsis leads to faster administration of antibiotics, which lowers patient mortality. A highly sensitive prediction tool can be helpful for early recognition and early resuscitation. To date, a variety of tools have been introduced and studied to predict outcomes, including the qSOFA score. However, there are still controversial results for the qSOFA score as a screening tool.

Recently, studies were performed in the US and Europe to determine the association between qSOFA scores and poor outcomes in patients with and without suspected infection in the ED. ${ }^{3,4}$ Based on these studies, we aimed to validate the ability of the qSOFA score to predict poor outcome of patients with suspected infection in the ED setting in South Korea.

\section{METHODS}

\section{Study design and setting}

This was a retrospective study at one urban emergency medical center in Seoul, Korea from January to December 2016. This study included adult patients ( $>18$ years) who presented to the ED with suspicion of infection, and for whom qSOFA score could be calculated. Electronic medical records and National Emergency Department Information System (NEDIS) data were reviewed for this period. The institutional review board of the Soonchunhyang University Seoul Hospital approved this study (2018-07-002). The requirement for informed consent was waived due to retrospective nature of the study.

\section{NEDIS data and triage}

NEDIS data include patient visit date, chief complaint, initial vital signs, reason for ED visit (illness-related or injury-related), mental status, method of transportation to the hospital, triage level, etc. NEDIS data is collected for all patients at the time of presentation to the ED, and triage by the Korean Triage and Acuity Scale (KTAS) occurs as soon as possible. The KTAS is a triage scale that was developed in Korea to recognize patients' severity. ${ }^{5}$ It includes simple history taking, vital signs (blood pressure, pulse rate, respiratory rate, temperature, and oxygen saturation) and mental status, as determined by a nurse who has completed a KTAS course and who has at least 1-year experience working in an ED. Ambulatory patients enter a triage room to collect triage data before entering the ED. Patients in serious condition who cannot ambulate come directly into the $E D$, and triage data is collected within a few minutes of patient arrival. 


\section{Patient selection}

Patients who were administered intravenous or oral antibiotics during the ED visit (including those who were prescribed antibiotics as discharge medication) were considered to have infection and were included in the study. We included every antibiotic available in our hospital. The exclusion criteria were patients younger than 18 years, patients who presented to the ED with injury, patients who presented to the ED for medical record copies or medical certification, and those in whom the qSOFA score could not be calculated.

\section{Measurements}

The qSOFA score was calculated by NEDIS data, and patients were grouped according to qSOFA score of $0,1,2$, and 3. Patient age, gender, admission rate, intensive care unit (ICU) admission rate, in-hospital mortality rate, length of hospital stay (LOS, days) from ED presentation to hospital discharge, and lactate level were compared among each qSOFA group. The calculation of in-hospital mortality included patients who were discharged as "expired," so those patients who were transferred to another health facility or discharged against medical advice were considered discharged alive and were not included in the calculation of in-hospital mortality. In addition, the lactate level of patients in the ED was compared for each qSOFA group to evaluate if lactate level increases as qSOFA increases, since lactate level and clearance rate are also known to affect the outcome of critically ill patients. ${ }^{6-8}$ The initial lactate level checked in the ED was used in the study. Usually, the lactate level was measured in those patients who were initially considered critically ill and were suspected to have infection. Lac- tate cut-off points of 2 and $4 \mathrm{mmol} / \mathrm{L}$ were used because these levels are commonly used in sepsis intervention. ${ }^{9}$

\section{Statistical analysis}

The chi-square test was used to compare categorical variables, and the Kruskal-Wallis test was used to analyze continuous variables. Values are given as the median and range. The linear-bylinear trend test was used to see the trend of qSOFA score compared with admission, ICU admission and mortality. Correlation analysis for qSOFA and LOS, qSOFA and lactate level was performed using the Spearman method. P-values $<0.05$ were considered statistically significant. To compare prognostic accuracy of qSOFA score and lactate level for in-hospital mortality, receiver operating characteristic curves and areas under the receiver operating characteristic curves (AUC) were calculated for qSOFA cutoff points and lactate levels of $2 \mathrm{mmol} / \mathrm{L}$ or higher and $4 \mathrm{mmol} / \mathrm{L}$ or higher. Analyses were performed with PASW Statistics ver. 18.0 (SPSS Inc., Chicago, IL, USA) and R ver. 3.3.1 ("AUC" package).

\section{RESULTS}

\section{Study subjects characteristics}

A total of 39,394 patients presented to the ED of Soonchunhyang University Seoul Hospital from January 2016 to December 2016. A total of 19,065 patients met the inclusion criteria. Finally, the study group included 2,698 patients, after excluding those with missing qSOFA scores and those without presumed infection (no antibiotics prescription in the ED) (Fig. 1).

Table 1 shows the number of patients, mean age, sex, and lac-

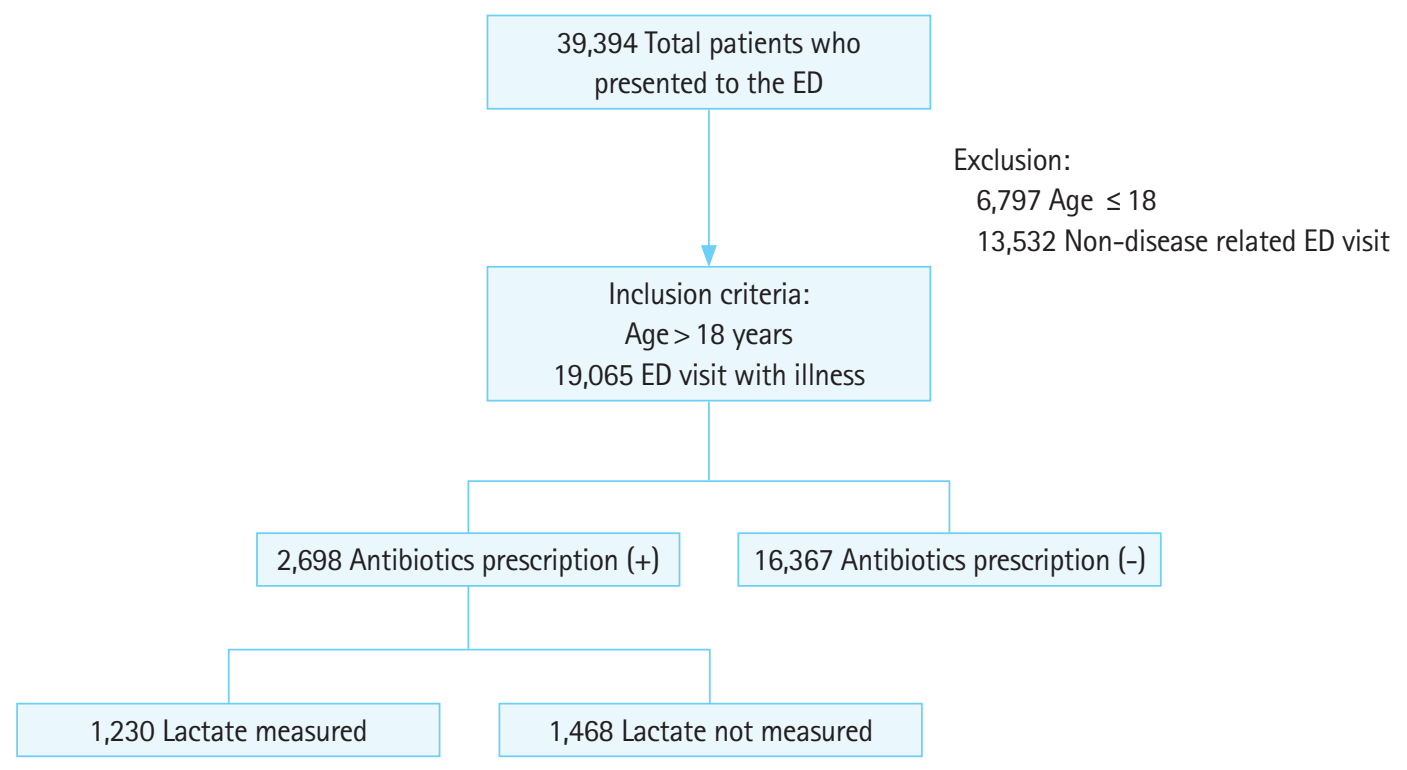

Fig. 1. Flowchart of patient selection. ED, emergency department; qSOFA, quick sequential organ failure assessment. 
Table 1. Study group characteristics

\begin{tabular}{|c|c|c|c|c|c|c|}
\hline & \multirow{2}{*}{ Total } & \multicolumn{4}{|c|}{ qSOFA score } & \multirow{2}{*}{ P-value } \\
\hline & & 0 & 1 & 2 & 3 & \\
\hline No. of patients & $2,698(100)$ & $1,665(61.7)$ & $829(30.7)$ & $171(6.3)$ & $33(1.2)$ & \\
\hline Mean age (yr) & $52.9 \pm 21.4$ & $49.3 \pm 20.2$ & $56.5 \pm 22.4$ & $65.0 \pm 19.8$ & $76.5 \pm 13.5$ & $<0.001^{\mathrm{a})}$ \\
\hline Female & $1,436(53.2)$ & $886(53.2)$ & $444(53.6)$ & $87(50.9)$ & $19(57.6)$ & $0.667^{b)}$ \\
\hline Lactate level measured & $1,230(45.6)$ & $680(38.4)$ & $437(52.7)$ & $123(71.9)$ & $30(90.9)$ & \\
\hline Mean lactate level $(95 \% \mathrm{Cl})(\mathrm{mmol} / \mathrm{L})$ & $2.2(2.11-2.37)$ & $1.8(1.72-1.96)$ & $2.3(2.07-2.50)$ & $3.2(2.58-3.73)$ & $6.2(4.08-8.41)$ & $<0.001^{\mathrm{c})}$ \\
\hline
\end{tabular}

Values are presented as number $(\%)$ or mean \pm standard deviation unless otherwise indicated. qSOFA, quick sequential organ failure assessment; $\mathrm{Cl}$, confidence interval.

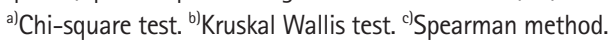

Table 2. Admission rate, ICU admission rate, mortality rate, and length of hospital stay of patients based on qSOFA score

\begin{tabular}{|c|c|c|c|c|c|c|}
\hline & \multirow{2}{*}{$\begin{array}{c}\text { Total } \\
(n=2,698)\end{array}$} & \multicolumn{4}{|c|}{ qSOFA Score } & \multirow{2}{*}{ P-value } \\
\hline & & $0(n=1,665)$ & $1(n=829)$ & $2(n=171)$ & $3(n=33)$ & \\
\hline Admission & $1,668(61.8)$ & $920(55.3)$ & $569(68.6)$ & $148(86.5)$ & $31(93.9)$ & $<0.001$ \\
\hline ICU admission & 238 (8.8) & $44(2.6)$ & 98 (11.8) & $72(42.1)$ & $24(72.7)$ & $<0.001$ \\
\hline In-hospital mortality & $134(5.0)$ & $37(2.2)$ & $53(6.4)$ & $30(17.5)$ & $14(42.4)$ & $<0.001$ \\
\hline Length of hospital stay (day) & $4(1-216)$ & $3(1-216)$ & $5(1-186)$ & $9(1-205)$ & $11(1-97)$ & $<0.001^{\text {b) }}$ \\
\hline
\end{tabular}

Values are presented as number (\%) or median (min-max).

ICU, intensive care unit; qSOFA, quick sequential organ failure assessment.

a) Linear-by-linear trend test. ${ }^{b)}$ Spearman method.
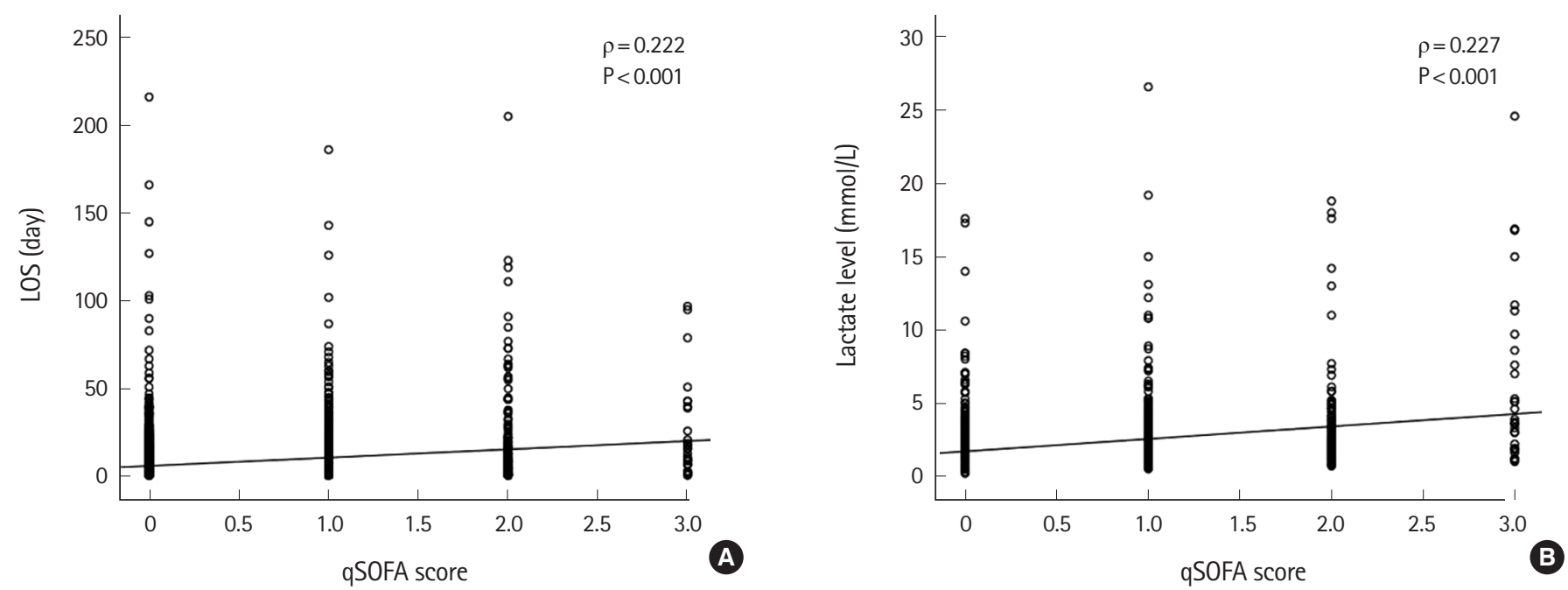

Fig. 2. Correlation between (A) quick sequential organ failure assessment (qSOFA) score and length of hospital stay (LOS), and (B) qSOFA score and lactate level.

tate level of patients in each qSOFA score group. The mean age was significantly different in each qSOFA score group and each group had a high proportion of women.

\section{Main results}

Table 2 shows the admission rate, ICU admission rate, in-hospital mortality rate, and LOS for patients in each qSOFA group. Notably, both admission and ICU admission rates, mortality rate, and
LOS (Fig. 2) all increased with increasing qSOFA score. Lactate levels were available for almost half of the patients, and the lactate level also increased with increasing qSOFA score (Table 1 and Fig. 2). The AUCs for predicting in-hospital mortality associated with qSOFA score, lactate $\geq 2 \mathrm{mmol} / \mathrm{L}$, and lactate $\geq 4 \mathrm{mmol} / \mathrm{L}$ were 0.719 (95\% confidence interval [Cl], 0.670 to 0.768$), 0.657$ (95\% Cl, 0.603 to 0.710$)$, and 0.632 (95\% Cl, 0.571 to 0.693$)$, respectively (Fig. 3). 


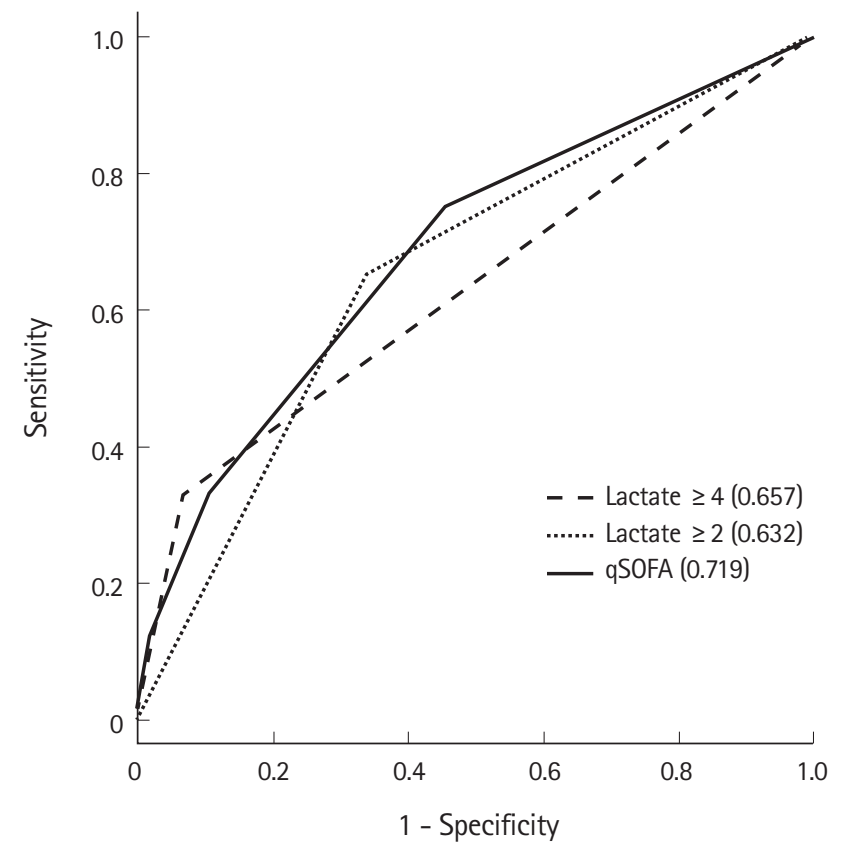

Fig. 3. Receiver operating characteristic curves for in-hospital mortality with lactate level $\geq 4 \mathrm{mmol} / \mathrm{L}$, lactate level $\geq 2 \mathrm{mmol} / \mathrm{L}$, and quick sequential organ failure assessment (qSOFA).

\section{DISCUSSION}

Identifying critically ill patients is an important task for emergency physicians. Our study showed positive performance of the qSOFA score as a screening tool for poor outcomes among ED patients with suspected infection in South Korea.

Previous sepsis guidelines used systemic inflammatory response syndrome (SIRS) to identify sepsis patients. SIRS is calculated from blood laboratory test results. For out-of-hospital or ED settings, however, waiting for laboratory reports may delay the resuscitation of critically ill patients. Therefore, the new sepsis guideline suggested use of the qSOFA score. Many studies have been performed after the release of this new guideline to confirm that the qSOFA score has significant predictive value in recognizing critically ill patients. ${ }^{3,49-14}$ To the best of the authors' knowledge, only a few studies of the qSOFA score have been conducted in South Korean-based EDs. ${ }^{15-17}$ Our study was the first to verify whether qSOFA could predict in-hospital mortality, admission rate, ICU admission rate, and LOS in South Korea in patients with suspected infection that present to the ED.

Our results showed that the patients' age increased with increasing qSOFA score. This is probably because older patients tend to have more serious (or advanced) presentation than younger patients. In addition to age, the qSOFA score was significantly associated with all outcomes, including admission rate, ICU admission rate, in-hospital mortality, and LOS, all of which increased with increasing qSOFA score. Furthermore, the qSOFA score had better performance for predicting poor outcome than lactate level. Therefore, our study showed positive results for qSOFA as a screening tool, and the qSOFA score might have strong predictive value in the ED for initial patient care.

Singer et al. ${ }^{3}$ studied qSOFA scores to predict in-hospital mortality in adult patients with or without suspected infection who visited an ED in the US. Like our study, theirs showed that qSOFA had a significant predictive value for in-patient mortality, admission, ICU admission, and hospital LOS. The mortality rate was $0.6 \%$, $2.8 \%, 12.8 \%$, and $25.0 \%$ for qSOFA scores $0,1,2$, and 3 , respectively. However, their study included noninfectious patients and selected infectious patient to whom only intravenous antibiotics were administrated. They used the Modified Early Warning System to calculate the QSOFA score, whereas we used a triage system, the KTAS, as a tool to screen patient severity, which allowed our study to calculate the qSOFA score during the initial patient encounter.

Freund et al. ${ }^{4}$ conducted a study in Europe to validate qSOFA as a mortality predictor. They included adult patients who visited the ED with suspected infection. The overall in-hospital mortality rate was $8 \%$, and patients with a qSOFA score of 2 or higher had a 24\% mortality. Their results showed that qSOFA performed better than both SIRS and severe sepsis criteria. The AUCs were 0.80 ( $95 \% \mathrm{Cl}, 0.74$ to 0.85 ) for qSOFA vs. 0.65 ( $95 \% \mathrm{Cl}, 0.59$ to 0.70 ) for SIRS and severe sepsis $(\mathrm{P}<0.001)$.

Rodriguez et al. ${ }^{9}$ conducted a study in the US to compare qSOFA to lactate levels for their ability to identify ED patients with sepsis with critical illness. This multicenter retrospective cohort study showed that the qSOFA criteria was better than lactate levels in predicting critical illness. The AUCs were 0.788 for qSOFA score and 0.763 for lactate, and the predictability of the qSOFA score was significantly higher $(P=0.0026)$.

A study conducted in South Korea compared SIRS and qSOFA score for predicting organ failure and in-hospital mortality in the ED. ${ }^{17}$ They compared the AUC for total of 1,009 patients. The predictive validity of qSOFA for in-hospital mortality was higher than that of SIRS (AUC $=0.733$ [95\% Cl, 0.64 to 0.83] vs. AUC $=0.599$ [95\% $\mathrm{Cl}, 0.51$ to 0.69$], \mathrm{P}=0.04$ ).

Hwang et al. ${ }^{16}$ studied the diagnostic performance of qSOFA for predicting 28-day mortality in critically ill septic patients in a Korean ED. They only included patients who were diagnosed with severe sepsis or septic shock. Contrary to our study, their outcome showed a poor diagnostic performance of qSOFA score (2 points or higher). This result might have been due to high illness severity of the study patients. In addition, the timing and methods of qSO- 
FA score variation might have caused these results.

There are some limitations in this study. First, owing to possible selection bias, the total number of study patients might have been over or under estimated. We defined study patients with suspected infection as those who had received intravenous or oral antibiotics in the ED, not by final diagnosis. Some patients without suspected infection in the ED but with a final diagnosis of infection-related disease might have received antibiotics after they were admitted to the hospital, and some might have stopped antibiotics after being admitted because they were ultimately diagnosed with a disease not related to infection. Nonetheless, we suppose that defining patients with suspected infection by their final diagnosis may result in selection bias as well. Second, our study included those who were transferred to other health care facilities or discharged against medical advice (173 of 2,698 patients, 6.4\%) as discharged alive; therefore, those who were transferred to hospice care or the moribund patients who were sent home were not included in our mortality count. Third, we did not check patient comorbidities, which might have affected the mortality cause. In addition, we did not review the hospital course, so whether infection was the cause of death was not fully determined. Even with these limitations, our study had a larger study group than previous studies that were performed in South Koreabased EDs.

In summary, this study analyzed whether the qSOFA score is reliable to predict poor outcome in patients with suspected infection in a Korean ED and showed that the qSOFA score was associated with poor outcome. Patients with higher qSOFA scores had higher admission rate, ICU admission rate, mortality rate, lactate level, and LOS. The qSOFA score had a better performance for predicting poor outcome than lactate level.

\section{CONFLICT OF INTEREST}

No potential conflict of interest relevant to this article was reported.

\section{REFERENCES}

1. Singer M, Deutschman CS, Seymour CW, et al. The third international consensus definitions for sepsis and septic shock (Sepsis-3). JAMA 2016;315:801-10.

2. Rhodes $A$, Evans LE, Alhazzani $W$, et al. Surviving sepsis campaign: international guidelines for management of sepsis and septic shock: 2016. Crit Care Med 2017;45:486-552.

3. Singer AJ, Ng J, Thode HC Jr, Spiegel R, Weingart S. Quick SOFA Scores predict mortality in adult emergency department pa- tients with and without suspected infection. Ann Emerg Med 2017;69:475-9.

4. Freund $Y$, Lemachatti $N$, Krastinova $E_{1}$ et al. Prognostic accuracy of sepsis-3 criteria for in-hospital mortality among patients with suspected infection presenting to the emergency department. JAMA 2017;317:301-8.

5. Park J, Lim T. Korean Triage and Acuity Scale (KTAS). J Korean Soc Emerg Med 2017;28:547-51.

6. Bhat SR, Swenson KE, Francis MW, Wira CR. Lactate clearance predicts survival among patients in the emergency department with severe sepsis. West J Emerg Med 2015;16:111826.

7. Gu WJ, Zhang Z, Bakker J. Early lactate clearance-guided therapy in patients with sepsis: a meta-analysis with trial sequential analysis of randomized controlled trials. Intensive Care Med 2015;41:1862-3.

8. Lee SM, Kim SE, Kim EB, Jeong HJ, Son YK, An WS. Lactate clearance and vasopressor seem to be predictors for mortality in severe sepsis patients with lactic acidosis supplementing sodium bicarbonate: a retrospective analysis. PLoS One 2015; 10:e0145181.

9. Rodriguez RM, Greenwood JC, Nuckton TJ, et al. Comparison of qSOFA with current emergency department tools for screening of patients with sepsis for critical illness. Emerg Med J 2018;35:350-6.

10. Amland RC, Sutariya BB. Quick Sequential [sepsis-related] Organ Failure Assessment (qSOFA) and St. John Sepsis Surveillance Agent to detect patients at risk of sepsis: an observational cohort study. Am J Med Qual 2018;33:50-7.

11. Bhattacharjee $P$, Edelson DP, Churpek MM. Identifying patients with sepsis on the hospital wards. Chest 2017;151:898-907.

12. Finkelsztein EJ, Jones DS, Ma KC, et al. Comparison of qSOFA and SIRS for predicting adverse outcomes of patients with suspicion of sepsis outside the intensive care unit. Crit Care 2017;21:73.

13. Haydar S, Spanier M, Weems P, Wood S, Strout T. Comparison of QSOFA score and SIRS criteria as screening mechanisms for emergency department sepsis. Am J Emerg Med 2017;35: 1730-3.

14. Wang JY, Chen YX, Guo SB, Mei X, Yang P. Predictive performance of quick Sepsis-related Organ Failure Assessment for mortality and ICU admission in patients with infection at the ED. Am J Emerg Med 2016;34:1788-93.

15. Cho YS, Moon JM, Chun BJ, Lee BK. Use of qSOFA Score in predicting the outcomes of patients with glyphosate surfactant herbicide poisoning immediately upon arrival at the emergency department. Shock 2019;51:447-52. 
16. Hwang SY, Jo IJ, Lee SU, et al. Low accuracy of positive qSOFA criteria for predicting 28-day mortality in critically III septic patients during the early period after emergency department presentation. Ann Emerg Med 2018;71:1-9.
17. Park HK, Kim WY, Kim MC, Jung W, Ko BS. Quick sequential organ failure assessment compared to systemic inflammatory response syndrome for predicting sepsis in emergency department. J Crit Care 2017;42:12-7. 